\title{
Data-driven Modelling of Thermal Solid Sorption Storage Systems
}

\author{
Harald Kirchsteiger ${ }^{*}$ (D) and Nayrana Daborer-Prado (D) \\ University of Applied Sciences Upper Austria, Energy Research Group ASIC, Wels, Austria
}

Received: 28 June 2021 / Received in final form: 19 July 2021 / Accepted: 19 July 2021

\begin{abstract}
An approach fofsr data-driven modelling of open sorption storage systems using zeolite as storage material is presented. The overall dynamic simulation model has the inflow air stream mass flow and absolute humidity as inputs and computes the outflow air temperature. The model is sub-divided into several components, where dynamic state space and process model identification techniques are applied. A comparison of the proposed modelling technique with simulated data from a validated model based on first principles shows that a reasonable accuracy - for a model application in temperature control systems design - can be obtained. It was found that using the proposed strategy, only a limited number of experiments are required, thus saving experimental time. Moreover, the computational requirements for a simulation using the proposed model are greatly reduced compared to a simulation model where differential equations discretised in time and space must be solved.
\end{abstract}

\section{Introduction}

As per December 2020, EU decided to cut down greenhouse-gas emissions by $55 \%$ (with respect to 1990) by the year 2030 [1]. Among the sectors responsible for a large share of the emissions are industry, electrical power generation, transportation, agriculture, and heating and cooling in commercial and residential buildings. In order to reach the ambitious goals, a significant emission reduction through technological innovation and political incentives in all the individual sectors is essential.

In the heating and cooling sector for commercial and residential buildings, technologies for renewable generation are available and well established. However, one major drawback is the intermittency and seasonal gap between high production in summer and high demand in wintertime of solar-driven energy production. An overproduction during periods of increased irradiation may not be required and is therefore wasted, while the undersupply must be covered by potentially non-renewable energy sources. Therefore, several techniques for long-term solar energy storage were analysed in the literature [2]. Among those techniques proposed, thermochemical storages are a very promising alternative [3] since they can significantly reduce the required space for the storage because of increased energy density of the material itself.

\footnotetext{
* e-mail: harald.kirchsteiger@fh-wels.at
}

Among thermochemical storages, sorption storage systems were gaining significant attention lately [4]. Many different combinations of two materials can form a sorption pair (for example silica gel and water vapor) and many different process concepts were proposed in the literature, see for example [5]. Arguably the most straightforward design of a sorption storage system is an open storage (in contrast to a closed storage under vacuum) as it was demonstrated for example in [6,7]. The basic and simplified working principle of sorption storages using the material combination water and zeolite is illustrated in Figure 1. The storage is charged by supplying heat to the sorption material which is getting dried in this process. This operating mode is called desorption. In a dry state, the sorption material can keep the stored energy without losses unless it is getting in contact with water-vapor. To discharge the storage, a controlled amount of water vapor is supplied to the material which in turn releases high amounts of heat. This operating mode is called adsorption. The whole process is repeatable all over again, however, thermomechanical stability of the zeolite might become an issue to consider [8]. Since there are no heat losses when kept in a dry state, seasonal storage becomes feasible [9].

Besides the properties of the material itself, the precise quantities of heat released per second depend mainly on the following three properties of the air stream which is guided through a storage compartment filled with the material: mass flow, inflow temperature and inflow humidity. The relation among those quantities is not a static one because it is well known that sorption materials have a limited 
storage capacity for water and moreover the uptake rate is nonlinear [10]. In order to use sorption storages in the best possible way in future renewable energy systems, mathematical models which numerically describe these relations are of great relevance, especially for modern model-based control algorithms and energy management systems.

While the literature on models for the adsorption process of single grains of material is extensive, the scientific literature on dynamical models of whole sorption storage systems is rather scarce. Models based on physical first principles were developed in [11-13]. A neural network model was identified in [14]. As with all physically motivated models, parameters have to be adapted to the particular system under observation through measurements.

The approach for modelling presented in this paper is starting straight away from measured data to obtain an input/output relation of temperature and humidity of the air flow through the storage. Instead of relying on material properties, all parameters are estimated from data and are not interpretable as physical quantities.

\section{Methodology}

Ultimately, the goal of this study is to derive a mathematical relation between the temperature $T_{I N}$ and

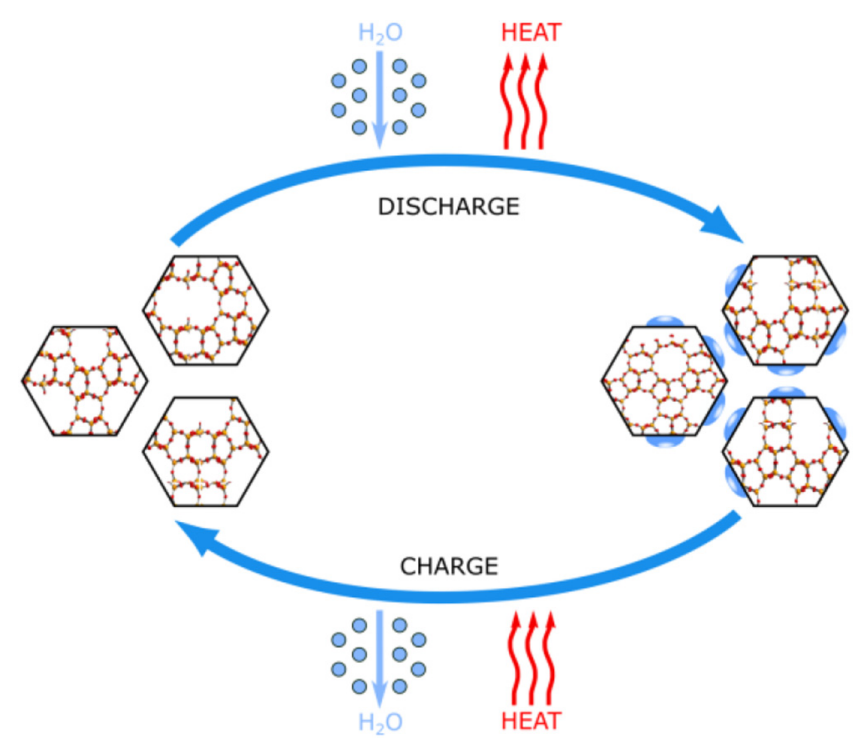

Fig. 1. Basic working principle of solid sorption storage. humidity $h_{I N}$ of an inflowing airstream into a fixed bed sorption storage schematically shown in Figure 2, and the temperature $T_{O U T}$ and humidity $h_{O U T}$ of the outflowing airstream. Another variable having a major influence on the output quantities is the mass flow $\dot{m}$ of the air. We are considering zeolite $13 \mathrm{X}$ as storage material in this study.

It is worthwhile to mention that from a systems theory point of view this system is a switching system (the dynamics are different in adsorption and desorption mode) and it is nonlinear (one nonlinearity being a saturation of the water uptake of storage material, which depends in level on the input variables).

The general strategy of the data-driven model development is subdivided into 4 stages:

- Obtain informative measurement data on a single adsorption experiment.

- Combine several experiments and develop a sub-model for the zeolite water saturation.

- Develop a sub-model for the output temperature.

- Combine the sub-models to an overall simulation model.

In the study presented here, measurement data was obtained from a physically motivated simulation model [12] which was already validated against a real-world storage setup. In this way, more information on the required amount of data and on experimental conditions can be learned without the need of expensive and timeconsuming lab measurements since the very beginning. An important side-result of the investigations presented here are guidelines for the exact procedure and setup of lab experiments which will make it possible to save considerable amounts of experimental time.

\subsection{Measurement data}

The three main influence factors on the storage behaviour which can be easily adjusted and measured are the temperature, absolute humidity, and mass flow of the incoming air stream. It is assumed the temperature $T_{I N}$ is always $20^{\circ} \mathrm{C}$, which is in agreement with the intended use of the storage as a heat generator for domestic buildings, driven by in-house ambient air. Additionally, a modest shift of this temperature would mainly result in a corresponding shift of the outflow temperature if the storage were properly insulated.

A single experiment conducted started with a welldefined initial water saturation of the storage material $x_{S}(0)$ given in $\mathrm{kg}$ water per $\mathrm{kg}$ zeolite dry mass. Two
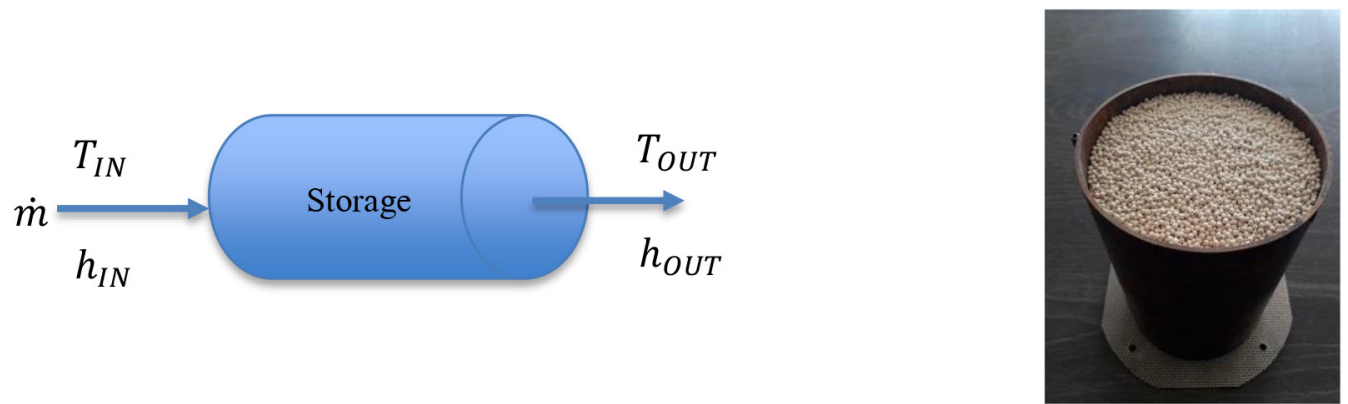

Fig. 2. Fixed bed reactor shown without additional appliances (right) and schematic view (left). 
Table 1. Symbols and values used in the simulation.

\begin{tabular}{lll}
\hline Symbol & Explanation & Value \\
\hline$m_{S}$ & Mass of storage material & $1 \mathrm{~kg}$ \\
$l_{S}$ & Length of storage & $0.33 \mathrm{~m}$ \\
$d_{S}$ & Diameter of storage & $0.09 \mathrm{~m}$ \\
$x_{S}(0)$ & Initial zeolite water saturation & $0.1 \mathrm{~kg} / \mathrm{kg}$ \\
$T_{S}$ & Data acquisition sample time & $10 \mathrm{~min}$. \\
$T_{I N}$ & Inflow air temperature & $20^{\circ} \mathrm{C}$ \\
$\dot{m}$ & Mass flow & {$[100 \ldots 400] \mathrm{kg} / \mathrm{h}$} \\
$h_{I N}$ & Inflow absolute humidity & {$[0.0043 \ldots 0.0087] \mathrm{kg} / \mathrm{kg}$} \\
$N_{\exp }$ & Total number of experiments & 30 \\
\hline
\end{tabular}

randomly chosen constant values inside of the ranges shown in Table 1 were attributed to the quantities $\dot{m}$ and $h_{I N}$. The experiment was stopped when the gradient of the water saturation was smaller than a pre-set threshold value $d_{x s}$. A total of $N_{\exp }$ experiments were conducted.

\subsection{Water saturation sub-model}

The water saturation sub-model maps the input quantities $\dot{m}$ and $h_{I N}$ to the intermediate quantity $x_{S}$. The general model structure assumption is a first order differential equation, which is given in state space form as

$$
\begin{gathered}
x_{k+1}=a x_{k}+\left(\begin{array}{ll}
b_{1} & b_{2}
\end{array}\right)\left(\begin{array}{l}
\dot{m} \\
h_{I N}^{*}
\end{array}\right), \\
x_{S}=c x_{k}+\left(\begin{array}{ll}
d_{1} & d_{2}
\end{array}\right)\left(\begin{array}{l}
\dot{m} \\
h_{I N}^{*}
\end{array}\right)
\end{gathered}
$$

where the coefficients $a, b_{1}, b_{2}, c, d_{1}, d_{2}$ are to be estimated from data and the state $x_{k}$ is of no particular interest. The observed nonlinearity between the inflow humidity and the maximum storage material saturation was compensated with a piecewise affine input transformation of the form

$$
h_{I N}^{*}=\alpha_{i} h_{I N}+\beta_{i}
$$

if $h_{I N}$ is within a region defined by $h_{I N, i-1}<h_{I N} \leq h_{I N, i+1}$. The coefficients $\alpha_{i}$ and $\beta_{i}$ are again estimated from data. For the estimation process, an increasing number of experiments was processed, with the aim to find the minimum number of required experiments for a reasonable model quality. The identification of the state space model was done with the prediction error method implemented in the System Identification Toolbox [15].

\subsection{Output temperature sub-model}

The model structure assumption for the temperature submodel is a parameter-varying first order process model with two inputs of the form

$$
T_{\text {OUT }}=\frac{K_{1}}{1+\tau_{1}(\dot{m}) s} u_{1}+\frac{K_{2}(\dot{m})}{1+\tau_{2}(\dot{m}) s} u_{2}
$$

where $K_{1}, \tau_{1}, K_{2}, \tau_{2}$ are the unknown parameters to be estimated from data and $s$ is the Laplace variable. Note that except for $K_{1}$, all parameters depend on the mass flow and are thus not constant parameters, making this model parameter varying. The estimation strategy for those parameters was a two-step procedure. First, individual process models were estimated on every single set of experimental data giving $N_{\exp }$ different estimates for the parameters $K_{1}, \tau_{1}, K_{2}, \tau_{2}$. While $K_{1}$ was found to be constant, the other parameters showed a strong dependency on the mass flow. Thus, second, polynomial dependencies of those parameters on the mass flow were estimated:

$$
\begin{gathered}
K_{2}(\dot{m})=K_{2,3} \dot{m}^{3}+K_{2,2} \dot{m}^{2}+K_{2,1} \dot{m}+K_{2,0} \\
\tau_{1}(\dot{m})=\tau_{1,3} \dot{m}^{3}+\tau_{1,2} \dot{m}^{2}+\tau_{1,1} \dot{m}+\tau_{1,0} \\
\tau_{2}(\dot{m})=\tau_{2,3} \dot{m}^{3}+\tau_{2,2} \dot{m}^{2}+\tau_{2,1} \dot{m}+\tau_{2,0} .
\end{gathered}
$$

The input quantity $u_{1}$ is derived from a linear regression of the inflow humidity with regards to the observed maximum outflow temperature $T_{O U T, \max }$

$$
u_{1}=\gamma_{1} h_{I N}^{*}+\delta_{1}
$$

where $\gamma_{1}, \delta_{1}$ are again parameters to be estimated, and the input quantity $u_{2}$ is the time derivative of the previously modeled storage material water saturation

$$
u_{2}=\frac{d}{\mathrm{~d} t} x_{S}
$$

\subsection{Overall simulation model}

The overall model is simply formed by combining all the sub-models and polynomial expressions defined above. Graphically, this is represented in Figure 3. For comparison purposes and evaluation, this model was compared to the simulation model presented in [12].

A flowchart showing the process of model estimation is given in Figure 4. The linear regressions were solved using standard Least-Squares approaches while the state space model and process model were estimated using 


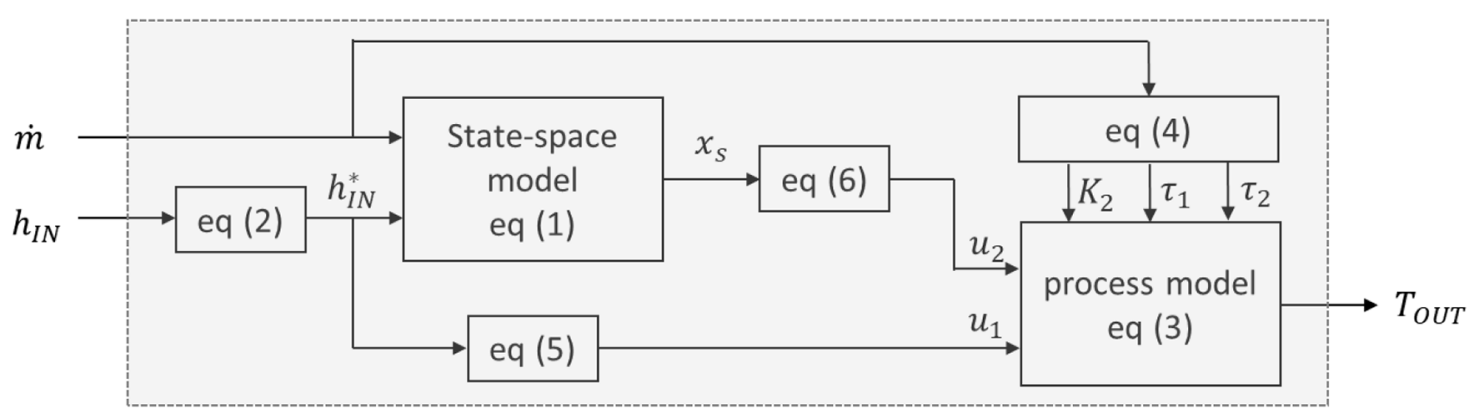

Fig. 3. Overall model structure.

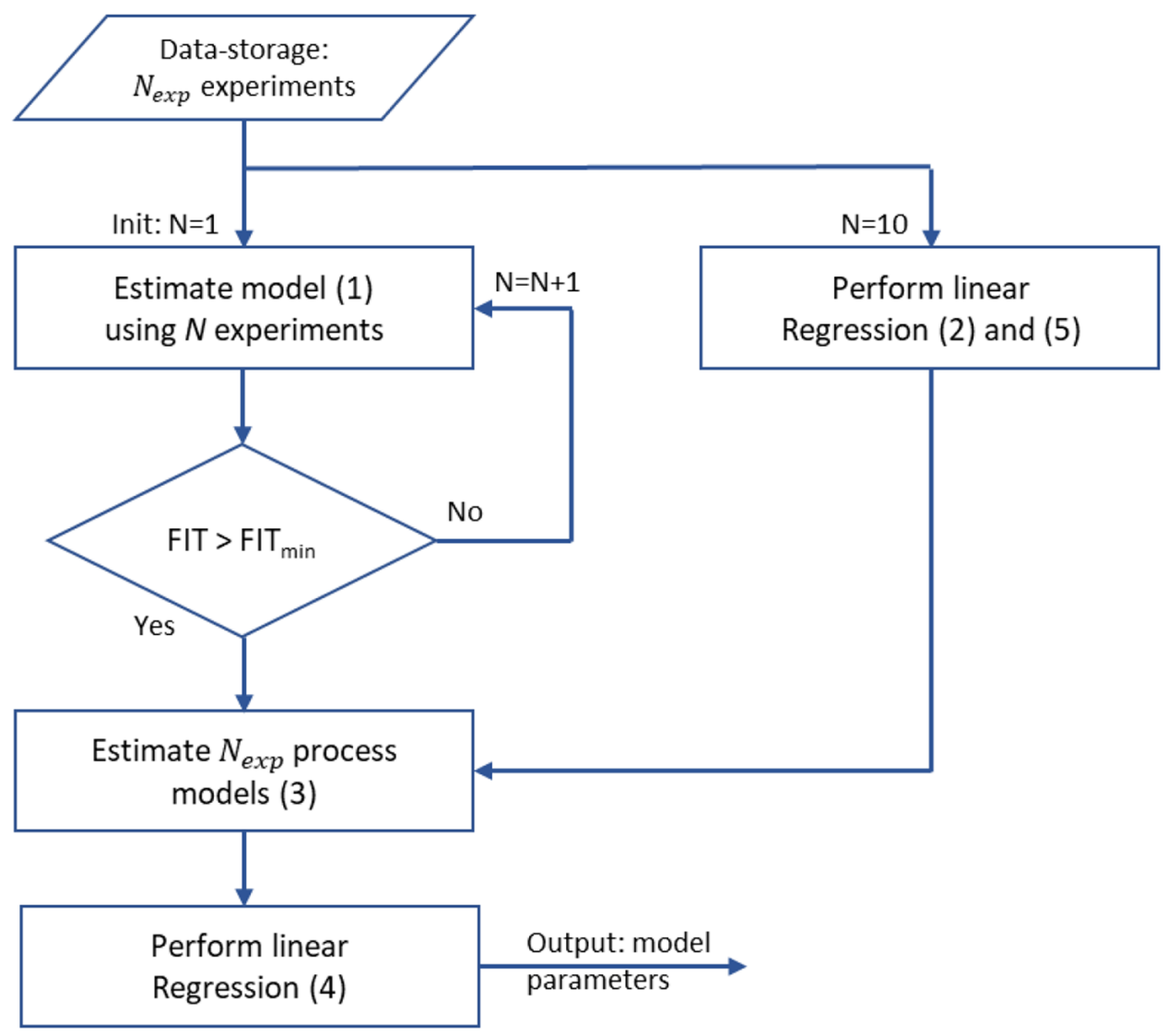

Fig. 4. Flowchart of the model estimation process.

sophisticated tools available from the System Identification Toolbox [15]. For the branching criterion, the average FIT value (see Eq. (7)) was computed on all available experiments, not only on the ones which were used for model parameter estimation.

\section{Data}

Measurement data was obtained from the simulation model [12] using the following set of parameters defined in Table 1. A total of $N_{\text {exp }}=30$ individual experiments were recorded, where each single one has a random value of $\dot{m}$ and $h_{I N}$ within the range as defined in the table. The recorded data also include information on the storage material saturation $x_{S}$, which can be obtained by gravimetric analysis in a lab.

\section{Results}

In this section graphical results, as well as numerical results using the FIT-value (7) as qualitative criterion how well an estimate $\hat{y}$ coincides with the signal $y$ to be modeled with mean value $\bar{y}$ are presented. A FIT value of $100 \%$ indicates perfect agreement between model and 
measurement while a value of $0 \%$ means the model is only able to correctly reproduce the mean value of the measurement. Also, negative values are possible.

$$
F I T=100\left(1-\frac{\|y-\hat{y}\|}{\|y-\bar{y}\|}\right)
$$

\subsection{Results on the water saturation sub-model}

The first $N=10$ experiments were used to determine the piecewise affine relation in equation (2). It was found that two linear pieces are sufficient to describe the relation, as it is show in Figure 5.

For the estimation of the state space model, sequentially an increasing number $N$ of experiments was used. Clearly, using only one dataset $N=1$ results in a good model fit for this particular case, but on the remaining 29 datasets the model performance will be not acceptable. In order to find a reasonable number of required datasets for

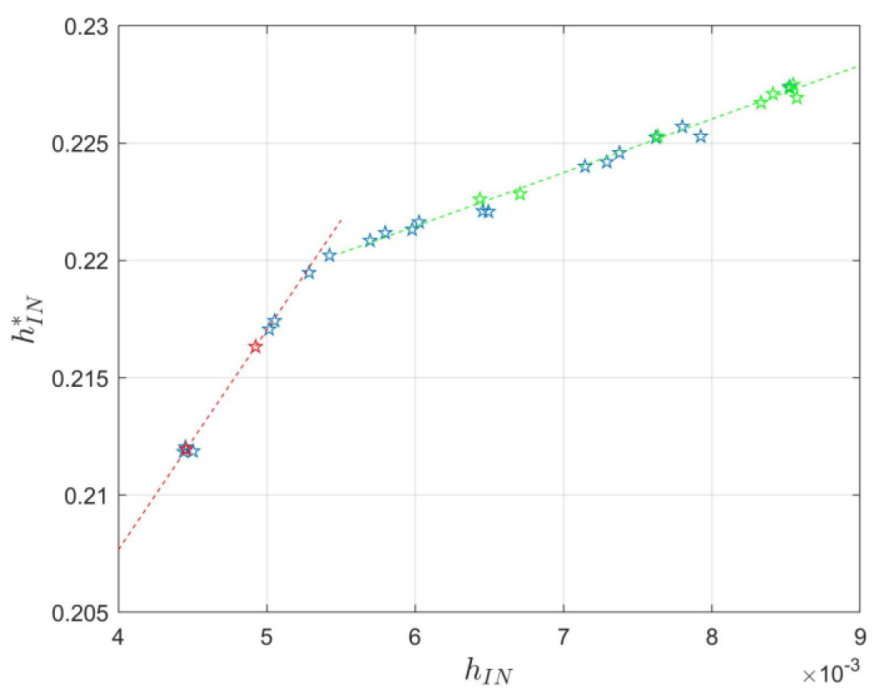

Fig. 5. Piecewise affine input transformation equation (2). Stars indicate measured data-points.
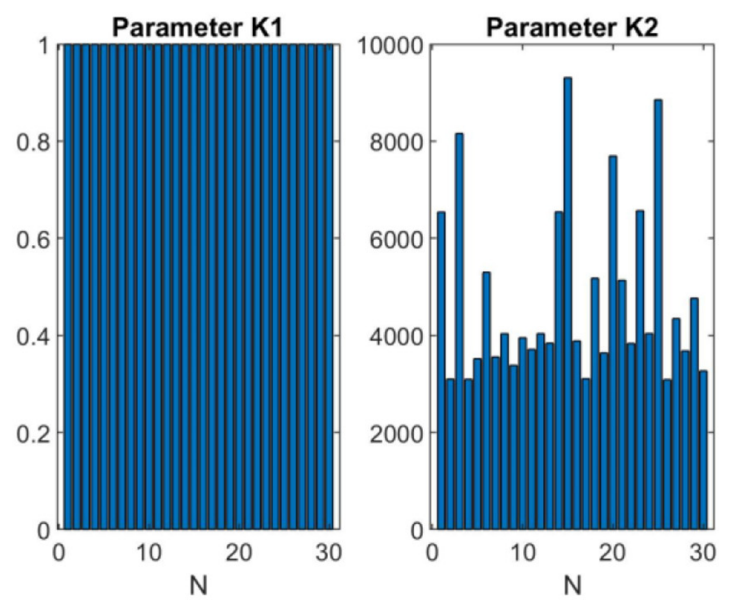

estimation, $N$ was sequentially increased, until the average fit value (7) on all the experiments used for identification did not change significantly anymore.

It was found that this is already the case for five experiments, where an average fit value of $88.99 \%$ is obtained for the datasets used for estimation and an average fit of $89.49 \%$ for the 25 independent datasets. Note that, in general, fit values on an independent validation dataset not used for model estimation are less than fit values on identification datasets. Using just one dataset for estimation, the fit is $93.86 \%$ compared to an average of 85.86 on the validation datasets.

\subsection{Results of the output temperature sub-model}

The linear regression model equation (5) was estimated to $\gamma_{1}=3296, \delta_{1}=20.35$. While the polynomial dependencies of the estimated model parameters of the linear parameter varying model structure equation (3) cannot be seen in Figure 6, they can be appreciated in Figure 7. It was found that a polynomial of third degree fits the data well. Note that the parameter $K_{1}$ is identified to be 1, regardless of the specific dataset used. Also note that once all the polynomials are fixed, model structure (3) is uniquely defined by the input air mass flow.

\subsection{Results of the overall simulation model}

All the estimated models were combined together (see Fig. 3) and simulated in parallel to the physically motivated simulation model from [12]. Once again, the input values for $\dot{m}$ and $h_{I N}$ were chosen randomly within the intervals previously defined in Table 1 . The final result of the output temperature can be seen in Figure 8 and shows a good agreement between the proposed data-based model and the reference signal.

\subsection{Remarks}

It has to be mentioned that extrapolation is critical for data-based models. The presented model is therefore only valid within the limits of the mass flow and humidity as
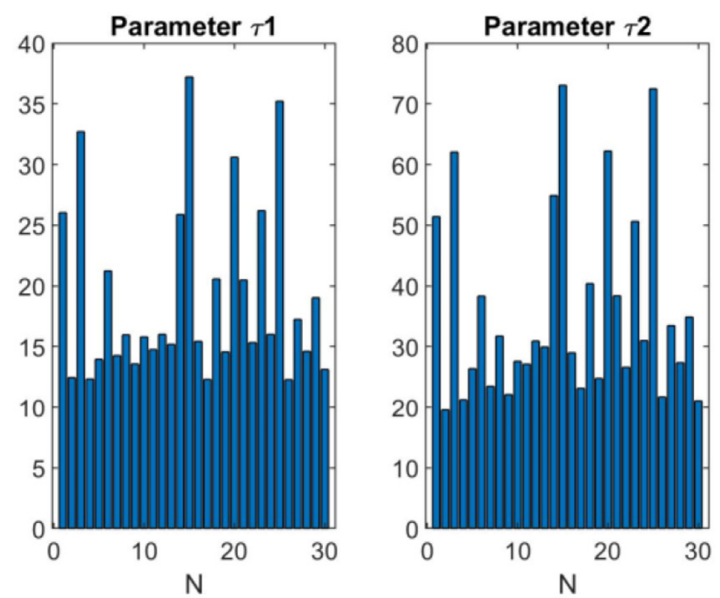

Fig. 6. Individually (using one dataset at a time) estimated model parameters of equation (3). 

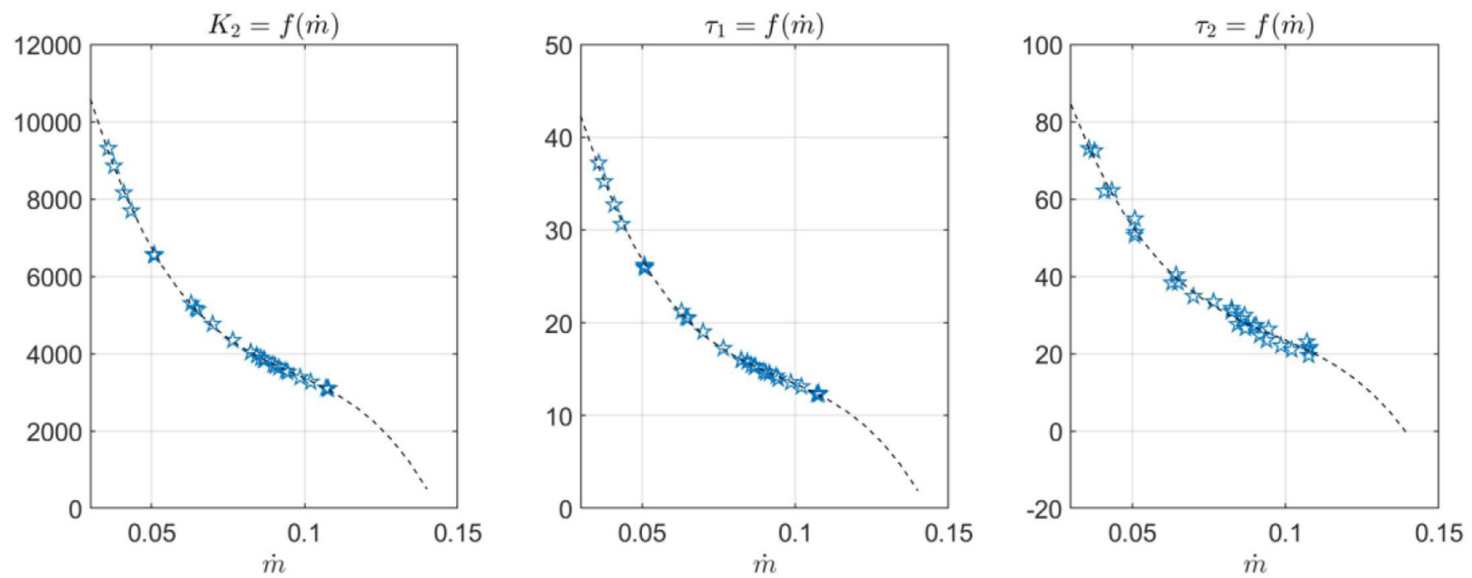

Fig. 7. Polynomial dependencies of the process model parameters in equation (3). Stars indicate measured data-points, dashed line is the estimated polynomial of 3rd order.
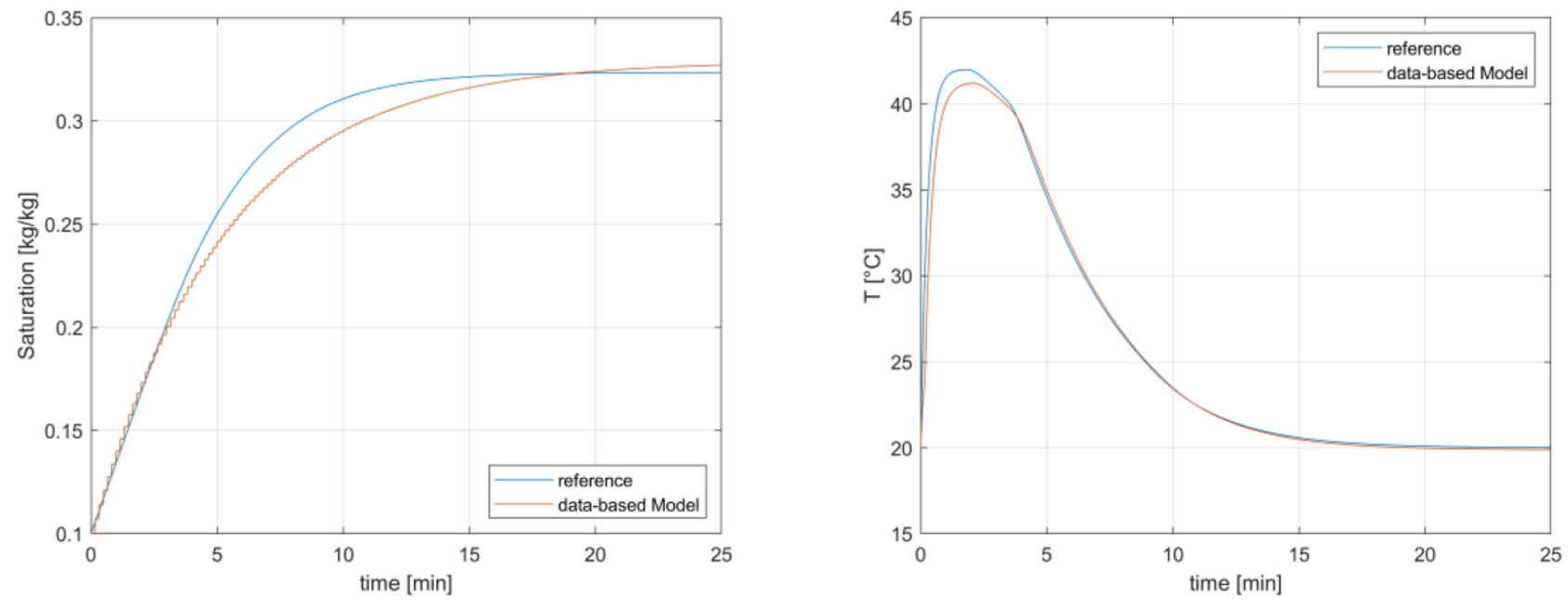

Fig. 8. Comparison between data-based model and simulation data as reference: zeolite saturation $x_{s}$ (left) and outflow air temperature $T_{O U T}$ (right).

defined in Table 1. It has to be mentioned also that the presented model is only considering the adsorption process. In the next step, the desorption process has to be analysed in detail. Also, the methodology is to be applied on the realworld experimental setup.

\section{Conclusion}

An entirely data-based modelling approach for solid sorption based thermal storage systems using zeolite as storage material was presented. The approach was tested against data from a previously validated simulation model. The end-results demonstrate a good agreement between the model output quantity which is the produced outflow temperature. Another finding is that only 5 experimental datasets are required to have sufficiently informative data that covers the entire region of interest. Due to its low computational requirements, the data driven model is well suited for implementation on a programmable logic controller used to control the operation of sorption storages. Moreover, the proposed model enables model-based control design approaches, for example model predictive control, which can significantly enhance the overall system performance. Compared to standard PID (proportional integral derivative) type approaches, they offer the possibility to inherently consider saturations and constraints and optimize the cyclic operation of the storage.

Acknowledgments. This project is financed by research subsidies granted by the government of Upper Austria.

\section{References}

1. European Commission, Stepping up Europe's 2030 Climate Ambition - Investigating in a Climate-Neutral Future for the Benefit of our People, Brussels, 17.09.2020 
2. P. Pinel, C.A. Cruickshank, I. Beausoleil-Morrison, A. Wills, A review of available methods for seasonal storage of solar thermal energy in residential applications, Renew. Sustain. Energy Rev. 15, 3341-3359 (2011)

3. G. Krese, R. Kozelj, V. Butala, U Stritih, Thermochemical seasonal solar energy storage for heating and cooling of buildings, Energy Build. 164, 239-253 (2018)

4. D. Aydin, S.P. Casey, S. Riffat, The latest advancements on thermochemical heat storage systems, Renew. Sustain. Energy Rev. 41, 356-367 (2015)

5. Y. Zhang, R. Wang, Sorption thermal energy storage: concept, process, applications and perspectives, Energy Storage Mater. 27, 352-369 (2020)

6. B. Zettl, G. Englmair, G. Steinmaurer, Development of a revolving drum reactor for open-sorption heat storage processes, Appl. Therm. Eng. 70, 42-49 (2014)

7. B. Zettl, H. Kirchsteiger, An open sorption heat storage application, in Proc. Int. Sustainable Energy Conference, Graz, Austria, 2018, pp. 605-611

8. F. Fischer, W. Lutz, J.C. Buhl, E. Laevemann, Insights into the hydrothermal stability of zeolite 13X, Micropor. Mesopor. Mater. 262, 258-268 (2018)
9. B. Fumey, R. Weber, L. Baldini, Sorption based long-term energy storage - process classification and analysis of performance limitations: a review, Renew. Sustain. Energy Rev. 111, 57-74 (2019)

10. D.D. Do, Adsorption Analysis: Equilibria and Kinetics (Imperial College Press, London, 1998)

11. G. Engel, S. Asenbeck, R. Köll, H. Kerskes, W. Wagner, W. van Helden, Simulation of a seasonal, solar-driven sorption storage heating system, J. Energy Storage 13, 40-47 (2017)

12. N. Daborer-Prado, Modeling and Simulation of an Innovative Domestic Sorption Storage System, M.S. Thesis, University of Applied Sciences Upper Austria, Wels, Austria, 2019

13. B. Mette, H. Kerskes, H. Drück, Experimental and numerical investigations of different reactor concepts for thermochemical energy storage, Energy Proc. 57, 2380-2389 (2014)

14. L. Scapino, H.A. Zondag, J. Diriken, C.C.M. Rindt, J. van Bael, A. Sciacovelli, Modeling the performance of a sorption thermal energy storage reactor using artificial neural networks, Appl. Energy 253, 113525 (2019)

15. The MathWorks, R2019a, System Identification Toolbox, Natick, Massachusetts, US

Cite this article as: Harald Kirchsteiger, Nayrana Daborer-Prado, Data-driven Modelling of Thermal Solid Sorption Storage Systems, Renew. Energy Environ. Sustain. 6, 27 (2021) 